\title{
Inelastic Neutron Scattering from Pressurized Ethane*
}

\author{
E. A. Straker \\ Department of Nuclear Engineering, The University of Michigan, Ann Arbor, Michigan
}

(Received 14 June 1965)

\begin{abstract}
Motions of the ethane molecule have been investigated at room temperature by inelastic neutron scattering techniques. Ethane targets were observed both in the gas and liquid phases by means of pressure variation. The University of Michigan four-rotor mechanical monochromator was used to obtain data at scattering angles of 90 and $22 \mathrm{deg}$ for neutron energies in the range 0.046 to $0.020 \mathrm{eV}$. Triple-axis-crystal spectrometer measurements were also made at 0.035 and $0.064 \mathrm{eV}$ as verification. These data indicate that there is no significant difference in the scattering properties of ethane in the gas and liquid state. Effects of the torsional motion of the two methyl groups about the $\mathrm{C}-\mathrm{C}$ bond can be seen in the forward-angle data. Comparison of experiment with theory indicates that a Krieger-Nelkin model which includes a harmonicoscillator representation of the torsional motion approximately predicts the observed data. The influence of multiple scattering on the final energy distribution has been observed and is discussed.
\end{abstract}

\section{INTRODUCTION}

$\mathbf{S}^{\mathrm{L}}$ LOW-neutron-scattering measurements have been made to examine the motions of the ethane molecule as the molecular density is varied, at fixed (room) temperature, from low-pressure gas to high-pressure liquid. It has been of particular interest to determine (1) if the translational, rotational, and internal torsional motions are influenced by the degree of condensation, and (2) if the optically-inactive torsional motion of the $\mathrm{CH}_{3}$ groups (lowest excitation energy of $275 \mathrm{~cm}^{-1}$ ) can indeed be observed by neutron interaction. A reasonably systematic measurement of the importance of multiple scattering has also been undertaken since this effect may obscure the motions sought.

Previous neutron investigations of ethane consist only of a total-cross-section determination by Melkonian ${ }^{1}$ in which the cross section was measured as a function of incident energy. Other molecules studied by inelastic neutron scattering in both the gas and liquid phase are methane $e^{2-8}$ and ammonia., ${ }^{4,8,10}$ Although there was a significant difference in the scattering properties of the two states of ammonia, little difference was found for

* Work supported in part by the National Science Foundation and in part by the U.S. Atomic Energy Commission.

${ }^{1}$ E. Melkonian, Phys. Rev. 76, 1774 (1949).

2 P. G. Randolph, R. M. Brugger, K. A. Strong, and R. E. Schmunk, Phys. Rev. 124, 460 (1961).

${ }^{3}$ J. A. Janik, J. M. Janik, J. Mellor, and H. Palevsky, J. Phys. Chem. Solids 25, 1091 (1964).

${ }^{4}$ F. J. Webb, Inelastic Scattering Neutrons Solids Liquids, Proc. Symp. 2nd Chalk River, Can., 1962 1, 457 (1963).

${ }^{5} \mathrm{~S}$. Hautecler and H. Stiller, Ref. 4, p. 423.

${ }^{6}$ K. Otnes, Bull. Am. Phys. Soc. 10, 42 (1965).

${ }^{7}$ B. A. Dasannacharya, G. Venkataraman, and K. U. Deniz, Inelastic Scattering Neutrons Solids Liquids, Proc. Symp. Bombay, India 1965, Paper 22.

${ }_{8}^{8}$ Y. D. Harker and R. M. Brugger, J. Chem. Phys. 42, 275 (1965).

${ }^{\circ}$ Phillips Petroleum Company, MTR-ETR Technical Branches Quarterly Report, IDO-16977, 31, 1963.

${ }^{10} \mathrm{~K}$. A. Strong, Y. D. Harker, and R. M. Brugger, J. Chem. Phys. 42, 1568 (1965). methane. In both of these measurements, the data for the liquid state were obtained at a lower temperature than for the gas in order to permit condensation. In the present experiment the temperature is held constant and the gas is liquefied by increasing the pressure; the liquid density was approximately 48 times that of the lowest gas pressure. By obtaining data at a constant temperature, the cross section for the two phases may be compared directly.

The data include inelastic scattering at an angle of $90 \mathrm{deg}$ for incident energies of $0.024,0.035,0.046$, and $0.064 \mathrm{eV}$, and at a scattering angle of $22 \mathrm{deg}$ and incident energy of $0.020 \mathrm{eV}$, for various gas target pressures between 40 and 530 psig. Liquid ethane was produced at pressures between 600 and $650 \mathrm{psig}$ and scattering data obtained at an angle of $90 \mathrm{deg}$ for energies of 0.024 and $0.035 \mathrm{eV}$.

Because of the translational and rotational degrees of freedom, the internal torsional motion does not appear as a sharp peak; only at forward scattering angles is there a separation of the torsional level from the translation-rotation maximum. At large scattering angles, excitation of the torsional mode contributes less importantly to the measured cross section and is harder to identify.

For comparison, theoretical calculations were made based on the Krieger-Nelkin model modified to include the torsional motion as a harmonic oscillator.

\section{EXPERIMENT}

As in all inelastic scattering experiments, the energy lost or gained by an incident neutron upon collision with a target molecule is required. The phased-rotor method developed by Egelstaf ${ }^{11}$ has been used for most of the present data. At The University of Michigan, a

11 P. A. Egelstaff, S. J. Cocking, and T. K. Alexander, Inelastic Scattering Neutrons Solids Liquids, Proc. Symp. Vienna, Austria, 1960,165 (1961). 
four-rotor mechanical monochromator ${ }^{12}$ has been built at the 2-MW Ford nuclear reactor. Two fiberglass rotors are used as rotating collimators to reduce the fast neutron background, and two magnesium-cadmium rotors of curved slit design form the burst of monoenergetic neutrons. Those neutrons which are scattered by the sample at an angle of $90 \mathrm{deg}$ are detected by a bank of seven ${ }^{6} \mathrm{LiF}-\mathrm{ZnS}$ scintillation detectors at a flight path of $2 \mathrm{~m}$ from the sample. A second detector bank, also at $2 \mathrm{~m}$, is located at $22 \mathrm{deg}$ and contains only three detectors to reduce the angular uncertainty in the scattering angle; this being $3.5 \mathrm{deg}$ for both detector banks. The detector pulses are time analyzed in a 256-channel time-of-flight analyzer, half of which is used to store the signal and half for background taken with a dummy in position; the target and dummy are alternated every 4 min. Eight-microsecond channel widths were used.

The beam is 1 by $1 \frac{1}{4}$ in. and there are approximately $3 \times 10^{5}$ neutron/min incident on the target at an energy of $0.024 \mathrm{eV}$. As a measure of the resolution, the elastic scattering peak of vanadium was observed and showed an energy full width at half-maximum of $0.0018 \mathrm{eV}$ at an incident energy of $0.024 \mathrm{eV}$. A typical scattering cross section is obtained in a 150 -h run.

Data reduction consisted of channel-by-channel background subtraction, correction for the energy dependence of the detector, and, for data which are to be compared with each other, a linear smoothing over nine data points (about one resolution full width).

To verify the rotor data and also provide a cross comparison of different instrumental methods, some measurements were also made at the University of Michigan triple-axis-crystal spectrometer. ${ }^{13}$ The raw data are corrected for second-order contamination and

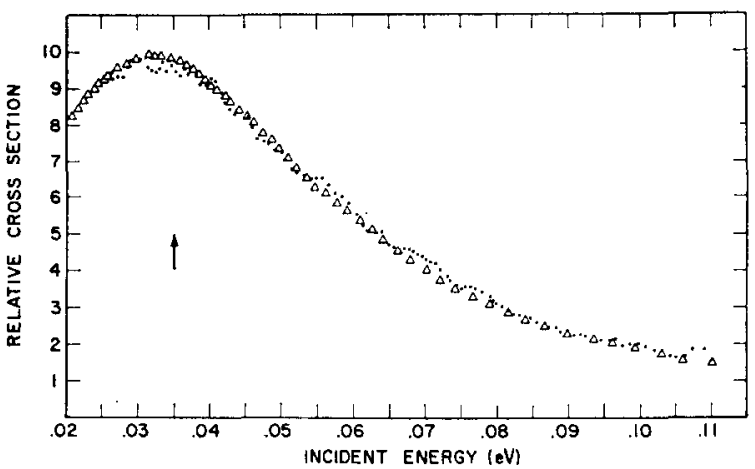

FIG. 1. Comparison of phased-rotor and crystal-spectrometer cross-section data for an energy of $0.035 \mathrm{eV}$ for gaseous ethane. $P=270$ psig; $\theta=90^{\circ} ; \triangle$ phased-rotor data; crystal data.

an incoherent background from the copper-analyzing crystals. The energy points reported in this case are closer spaced and averaging has been performed over adjacent points. Figure 1 shows the good agreement between comparable data obtained by the two different methods; the time-of-flight data have been transformed to a cross section of variable incident energy by using detail balance and by the time of flight to energy relationship.

The high-pressure target holder, ${ }^{14}$ see Fig. 2, is a cylinder of 6061-T6 aluminum $12 \mathrm{in.}$ long and $3.5 \mathrm{in.}$ in diameter. The center section has a wall thickness of $0.080 \mathrm{in}$., and the cylinder is sealed at each end with an $\mathrm{O}$ ring and threaded end plug. The scattering volume is defined by the inside wall of the aluminum cylinder and a cadmium-covered insert. For each set of data the insert is positioned to provide a gas thickness which has the desired transmission calculated from the meas-

FIG. 2. High-pressure target holder.

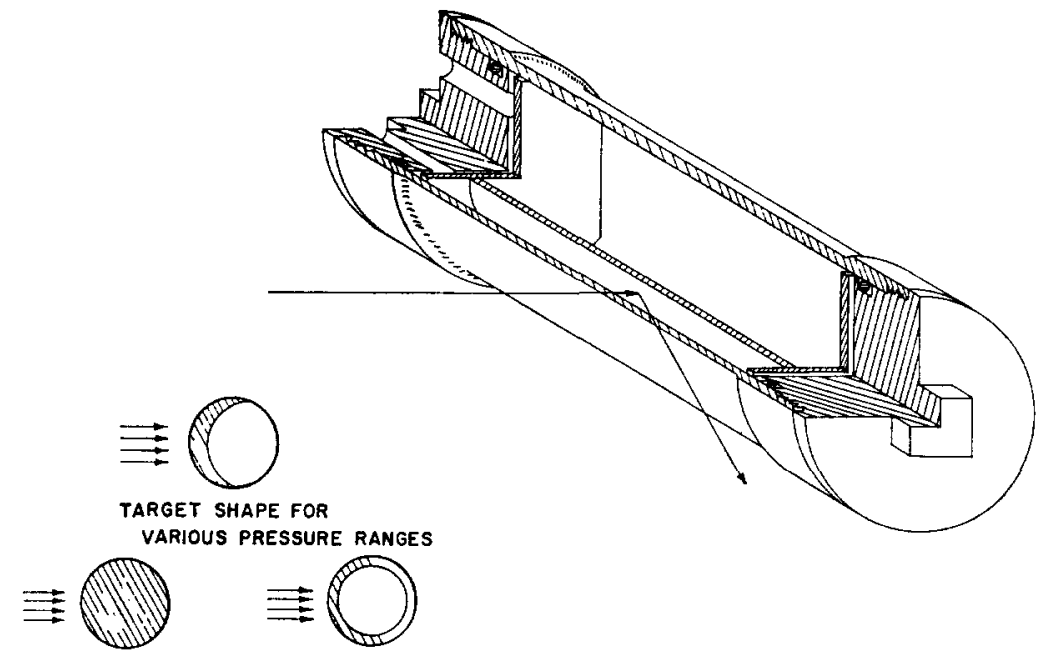

${ }^{12}$ P. F. Zweifel, "Neutron Cross Sections and Spectra Studies," University of Michigan, Report 03712-5-P 1964.

13 J. S. King and J. L. Donovan, Bull. Am. Phys. Soc. 9, 623 (1964).

${ }^{14}$ E. Straker, D. H. Vincent, and J. S. King, Bull. Am. Phys. Soc. 10, 21 (1965). 


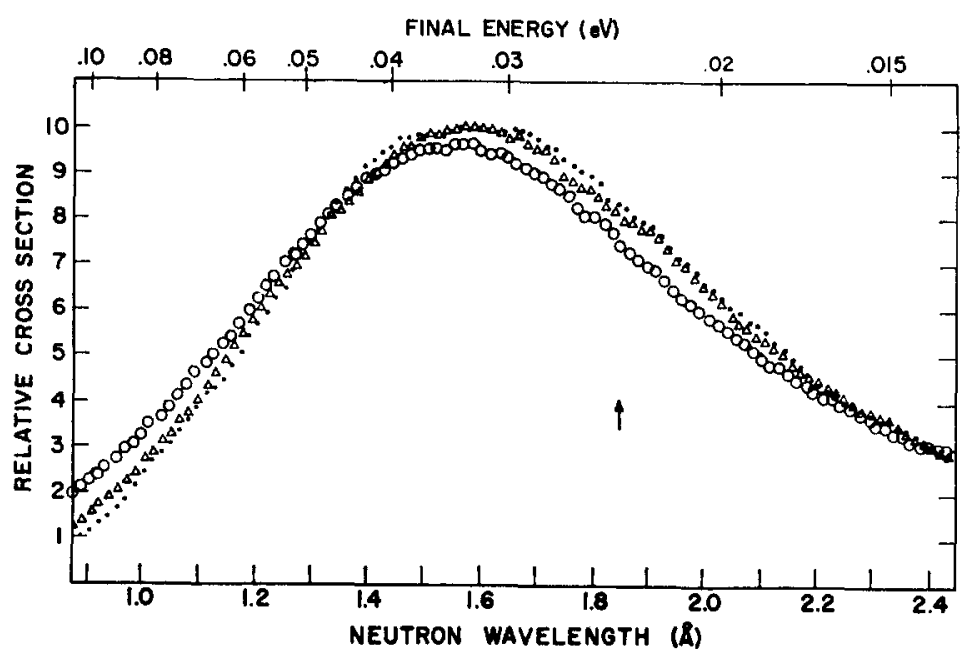

FIG. 3. Effects of multiple scattering at an angle of $90 \mathrm{deg}$ for an incident energy of $0.024 \mathrm{eV}$ for gaseous ethane. $P=500 \mathrm{psig}, \theta=90^{\circ} ; E_{i}=0.024 \mathrm{eV}$; transmission $90 \%, \triangle$ transmission $75 \%, O$ transmission $50 \%$.

ured cross section. 1 These thicknesses range from 3.0 to 0.050 in. depending on the target density. At high densities, scattered neutrons are observed in reflection from the horizontal cylinder. For the low pressure, forward angle measurements, a cylinder 2 in. in diameter with 0.030 -in.-thick windows was used in transmission. Purity of the ethane gas was greater than $99 \%$ with principal impurities being other hydrocarbons. The temperature was not controlled but remained at $298^{\circ} \mathrm{K}$ with maximum variations of $4^{\circ}$.

To investigate the effect of multiple scattering, data for several target thicknesses were obtained at a scattering angle of $90 \mathrm{deg}$ for $0.024-\mathrm{eV}$ neutrons. The relative scattering cross section for targets of $90 \%$, $75 \%$, and $50 \%$ transmission are shown in Fig. 3 ; the curves have been normalized to equal area. It is evident that, while there is little difference for the $90 \%$ and $75 \%$ targets, distortion in the data occurs for the $50 \%$ target. Similar results, Fig. 4(a), were obtained at the forward angle of $22 \mathrm{deg}$ and $0.020 \mathrm{eV}$. To minimize distortion due to multiple scattering, targets should have a transmission of at least $75 \%$, and $90 \%$ targets would be preferable when compatible with intensity requirements.

One of the problems inherent in observing pressurized gases is scattering from the target holder. To minimize this effect, an identical dummy holder was used to obtain background. However, due to crystalline differences in the two holders, a net aluminum contribution to the measured cross section may be obtained. This contribution results from differences in shape and amplitude of the elastic scattering from the holders. Evidence of this is seen in some of the phased-rotor data near the arrow indicating the incident energy. In the crystal data a different problem arises. Because the incident energy is varied, there are several Bragg conditions for 90-deg scattering satisfied for planes in the aluminum holder; thus target-holder effects are noted in several places. This is a disadvantage inherent in this crystal spectrometer system when relatively thick target containers are required.

\section{PRESSURE EFFECT}

The results of varying the pressure from 150 - to 500-psig gas and to 650-psig liquid are shown in Fig. 5 (a). The incident energy was $0.024 \mathrm{eV}$, the scattering angle $90 \mathrm{deg}$, and the relative cross-section differential in angle and wavelength is given as a function of the neutron wavelength. The calculated target transmissions were $75 \%$. The data have been smoothed over nine adjacent data points to facilitate general shape comparison.

It is evident that no apparent change in the cross section occurs as the gas is condensed. The differences near the incident energy are believed to be due to the aluminum target holder. Crystal spectrometer data at 0.035 and $0.064 \mathrm{eV}$ also indicate no change in the cross section upon condensation of the gas; see Figs. 5(b) and $5(\mathrm{c})$. The range here was extended from 70-psig gas to 600 -psig liquid producing a density change of a factor of 48 , and still there is no detectible change in shape for the cross section. It would have been valuable to extend the pressure measurements to the lowmomentum-transfer (small-scattering-angle) region, but the target design restricted the small-angle experiments to low pressure.

\section{TORSIONAL MOTION}

At the higher energy and scattering angles, the contribution from excitation of the torsional motion is small compared to the translation-rotation peak in the cross section, and its effect is to slightly shift the peak in energy. Measurements were, therefore, made at low incident energy $(0.020 \mathrm{eV})$ and forward scattering angle ( $22 \mathrm{deg}$ ) in order to separate the torsional component. The differential cross section is shown without smoothing in Fig. 4(b). The transmission was calculated to be $86 \%$ and agreed well with a transmission meas- 
FIG. 4. Phased-rotor data for the scattering cross section of ethane at an angle of $22 \mathrm{deg}$ and $0.020 \mathrm{eV}$ incident energy. (a) Multiple-scattering data normalized to equal area. Transmission $86 \%, P=55$ psig; $\triangle$ transmission $67 \%, P=155$ psig. (b) Comparison of unaveraged data with theory. Transmission $86 \%$; theory with oscillator; - - theory without oscillator; $P=55$ psig.

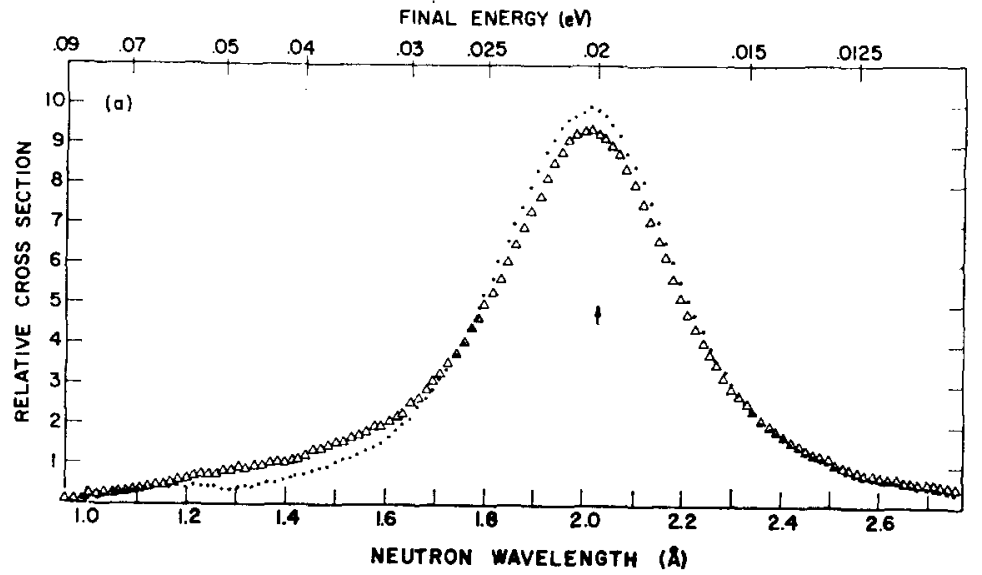

(a)

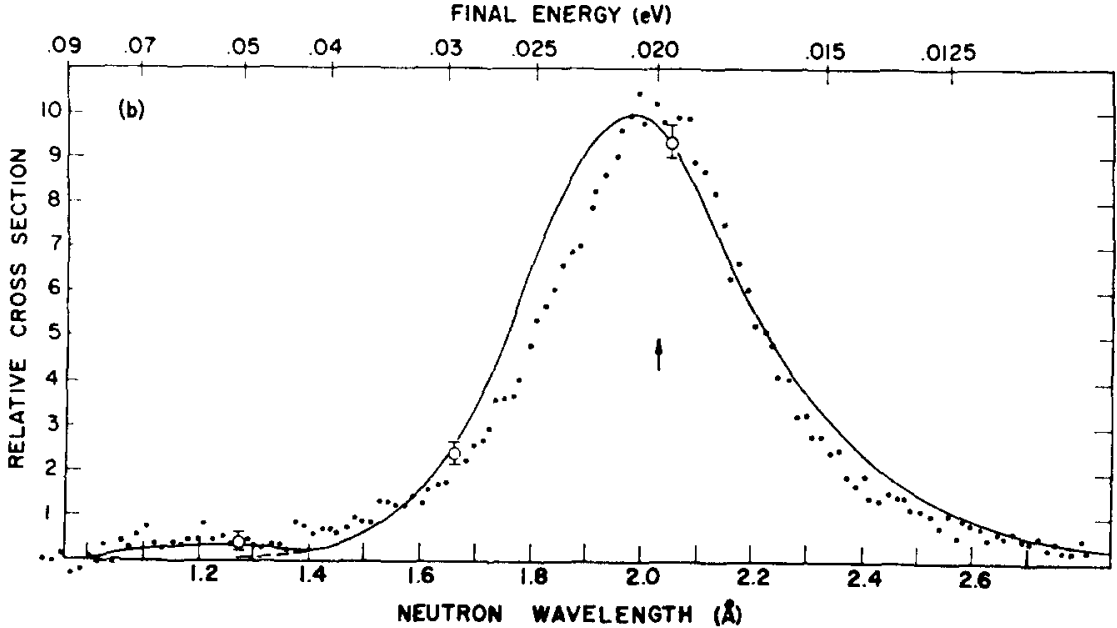

(b) urement. Although the counting statistics are poor, the positive contribution in the region from 1.0 to $1.4 \AA$ is believed to be due to the torsional excitation. The data are distributed about an energy transfer of approximately $0.034 \mathrm{eV}$ in good agreement with specific-heat data. ${ }^{15}$ Distortion of the forward-angle data due to multiple scattering is noted in Fig. 4(a). It is believed, however, that multiple-scattering effects in the thintarget data of Fig. 4(b) are insignificant.

\section{THEORY}

The expected energy distribution of scattered neutrons was calculated based on the assumption that rotations, vibrations, and translations could be treated as independent motions. Furthermore, it is assumed that there are no significant interactions between molecules regardless of the target density. Based on the Krieger-Nelkin model, ${ }^{16}$ translations are treated quantum mechanically as an ideal gas, and rotations are

${ }^{15}$ G. B. Kistiakowsky, J. R. Lacher, and F. Stitt, J. Chem. Phys. 7, 289 (1939).

${ }^{10}$ T. J. Krieger and M. S. Nelkin, Phys. Rev. 106, 290 (1957). treated classically with a continuum of energy states. The torsional motion about the $\mathrm{C}-\mathrm{C}$ bond is assumed to be a vibration and is treated as a harmonic oscillator; all other vibrations are treated as oscillators in their ground state. Averaging of the resulting cross section over all molecular orientations is performed using the Krieger-Nelkin technique of replacing functions of the Euler angles by their average value. Only direct scattering by the hydrogen and carbon atoms is included.

Based on the above assumptions, the cross section is given by

$$
\begin{aligned}
\sigma\left(E_{\imath} \rightarrow E_{f}, \theta\right)=\left(\frac{1}{4 \pi k T}\right)^{\frac{1}{3}} \sum_{\nu} a_{\nu}{ }^{2}\left(\frac{E_{f}}{E_{i} E_{R_{\nu}}}\right)^{\prime} \\
\quad \times \exp \left[-\left(\hbar \kappa^{2} C_{\nu} / 6 \omega\right) \operatorname{coth}(\hbar \omega / 2 k T)-\hbar^{2} \kappa^{2} \gamma\right] \psi,
\end{aligned}
$$

where

$$
\begin{gathered}
\psi=\sum_{n=-\infty}^{\infty} I_{n}\left(\frac{\hbar \kappa^{2} C_{\nu}^{2}}{6 \omega} \operatorname{csch} \frac{\hbar \omega}{2 k T}\right) \\
\times \exp \left[-(n \hbar \omega / 2 k T)-\left(E_{f}-E_{i}+E_{R_{\nu}}-n \hbar \omega\right)^{2} / 4 E_{R \nu} k T\right], \\
\quad E_{R_{\nu}}=\left(m / M_{\nu}\right)\left[E_{f}+E_{i}-2\left(E_{i} E_{f}\right)^{\frac{1}{2}} \cos \theta\right],
\end{gathered}
$$




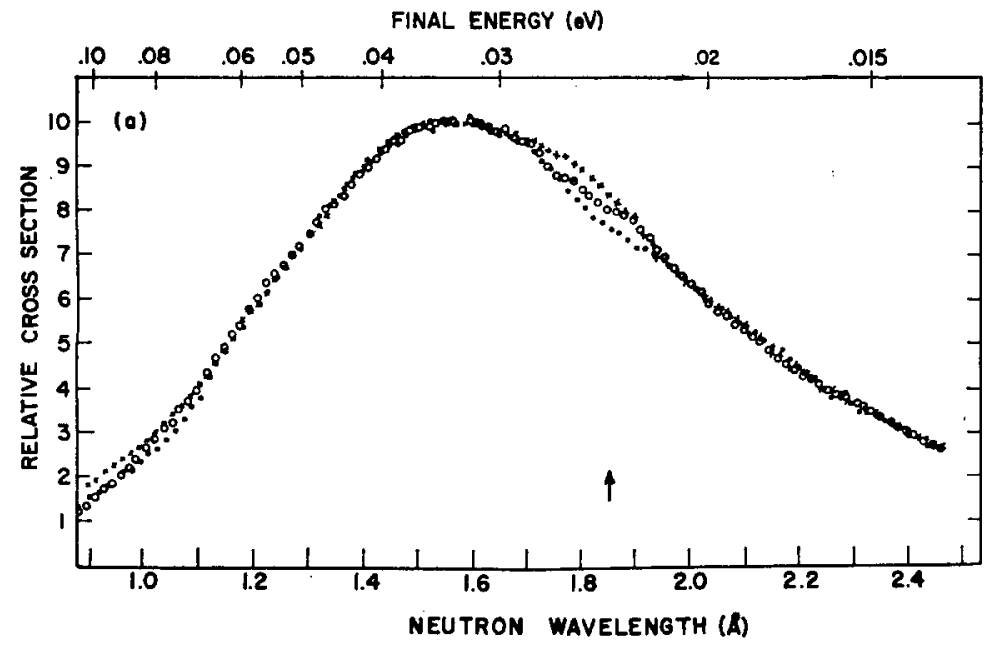

(a)

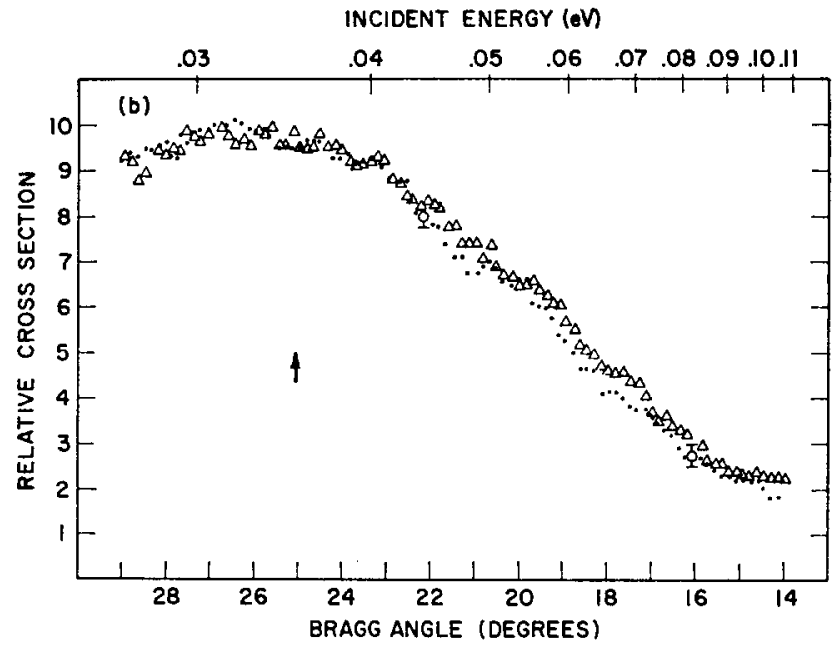

(b)

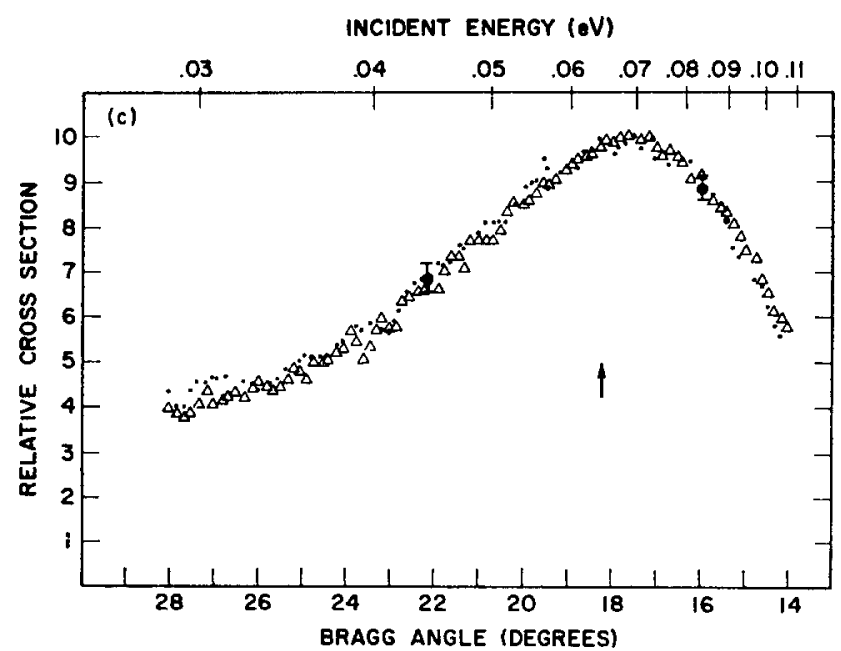

(c)

FIG. 5. Comparison of relative scattering cross section for various pressures of gaseous and liquid ethane at an angle of $90 \mathrm{deg}$ and temperature of $298^{\circ} \mathrm{K}$. Transmissions are $75 \%$. (a) Phased-rotor data $\cdot E_{i}=0.024 \mathrm{eV} ; 150$-psig gas, $\bigcirc 500$-psig gas, $\times$ 650-psig liquid. (b) Crystal data: $E_{f}=$ $0.035 \mathrm{eV} ; 70$-psig gas, $\triangle 600$-psig liquid. (c) Crystal data: $E_{f}=0.064 \mathrm{eV} ; 0$ 70-psig gas, $\triangle$ 530-psig gas. 


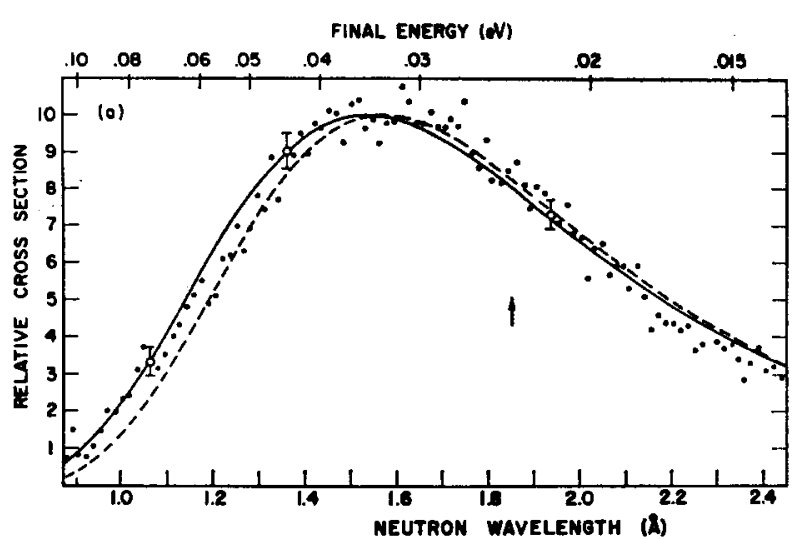

(a)

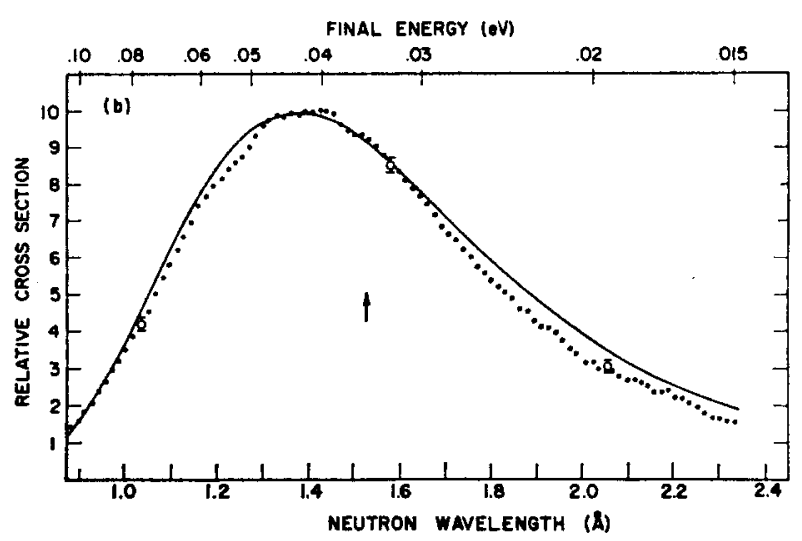

(b)

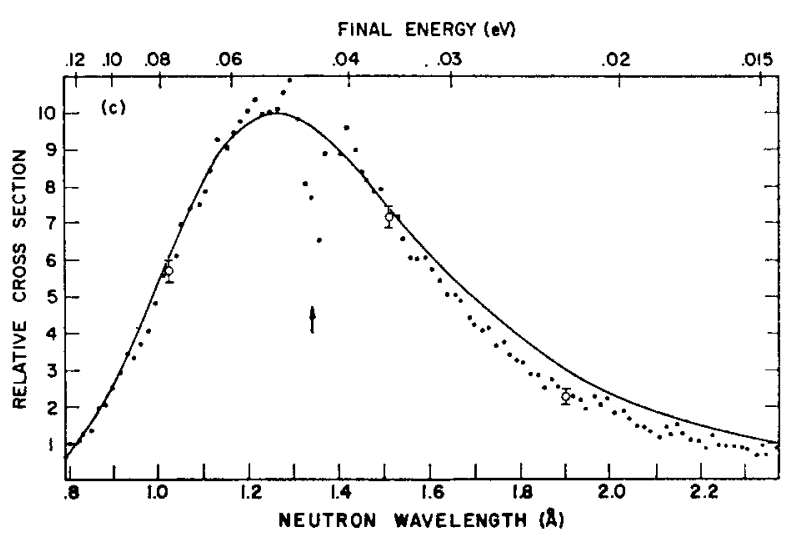

(c)

FIG. 6. Comparison of scattering cross section for gaseous ethane with theoretical calculations for $90 \mathrm{deg}$ scattering angle. Unaveraged phased-rotor data for various incident energies. -Theory with oscillator; ...--theory without oscillator. (a) $E_{i}=0.024 \mathrm{eV}$, transmission $90 \%, P=40$ psig. (b) $E_{i}=0.035 \mathrm{eV}$, transmission $75 \%, P=270$ psig. (c) $E_{i}=0.046$, transmission $75 \%$, $P=140$ psig.

$$
\gamma=\frac{1}{6} \sum_{\lambda}\left(C_{\nu}^{\lambda 2} / \hbar \omega_{\lambda}\right)
$$

and

$\begin{array}{ll}E_{i} & \text { Incident neutron energy } \\ E_{f} & \text { Final neutron energy } \\ \kappa & \text { Momentum transfer } \\ k T & \text { Target temperature } \\ \hbar \omega & \text { Torsional level spacing } \\ a, & \text { Scattering length for } \nu \text { th atom } \\ M, & \text { Sachs-Teller mass for } \nu \text { th atom } \\ m & \text { Neutron mass } \\ \theta & \text { Scattering angle } \\ C, & \text { Amplitude of } \nu \text { th atom during torsional oscil- } \\ & \quad \text { lation } \\ \gamma & \text { Vibrational parameter. }\end{array}$

For ethane the Sachs-Teller masses for hydrogen and carbon are $7.35 m$ and $20.5 m$, respectively. The amplitude of displacement of the hydrogen atom during the torsional motion is obtained from the normalization condition $^{16}$

$$
\sum_{p} m_{\nu} C_{\nu}^{2}=1
$$

Since only the hydrogen atoms move, $C_{\mathrm{H}^{2}}{ }^{2}=1 / 6 m_{\mathrm{H}}$. The vibrational parameter was calculated as $2.3 / \mathrm{m}$ from the work of Hansen and Dennison. ${ }^{17}$ Thus zeropoint vibrational effects are not negligible.

The solid curves in Figs. 4(b), 6(a), 6(b), and 6(c) were calculated from Eq. (1) with the oscillator excitation included. The dashed curves in Figs. 4(b) and 6(a) were calculated from the same equation but with only the $n=0$ term of the sum, that is, no excitation of the torsional motion. Thus, the difference between the solid and dashed curves illustrates the calculated contribution of the torsional excitation to the cross section.

Classical treatment of rotations appears to be satisfactory for the 90 -deg scattering angle (large momentum transfer). However, it is probable that the discrepancy with the data points for the forward angle in the immediate wings of the peak, Fig. 4(b), is due to failure of the classical model of rotations for the smaller momentum transfers. In the region of the first oscillator level, approximately 1.0 to $1.4 \AA$ in Figs. 4 (b) and $6(\mathrm{a})$, the theoretical amplitude of the oscillator contribution is too small at the forward angle, yet too

${ }^{17}$ G. E. Hansen and D. M. Dennison, J. Chem. Phys. 20, 313 (1952). 
large at 90 deg. Although this disagreement may be due to normalization of the theoretical calculation to the peak in the observed cross section, it may also be due to the approximate representation of the torsional motion as a harmonic oscillator. To be rigorous, the torsional motion should be treated as a hindered internal rotation. Recent work ${ }^{18}$ has shown that this motion may be strongly coupled to free rotations, contrary to what has been assumed here.

\section{CONCLUSIONS}

The 22-deg scattering data are believed to definitely establish the presence and importance of the torsional motion, although it is obscured at $90 \mathrm{deg}$. There is no observable change in the cross section with gas condensation for density variations from 7 to $340 \mathrm{mg} / \mathrm{cc}$. Thus, free rotations must exist in liquid ethane as has

${ }^{18}$ J. D. Erickson (private communication). been previously shown to be the case for methane. The data are approximately fit by a Krieger-Nelkin model with discrepancies at small momentum transfers being attributed to the classical treatment of rotations. Disagreements in the region of the oscillator excitation are believed due to the approximate treatment of the torsional motion in which it is decoupled from free rotations and treated as a harmonic oscillator. These disagreements cannot be attributed to multiple scattering since it is seen that distortions due to multiple events are small for the data which are compared with theory.

\section{ACKNOWLEDGMENTS}

The author is indebted to Dr. J. S. King for many helpful discussions involving the experiment and its interpretation, to J. D. Erickson for discussions on the theoretical model, and to Dr. D. H. Vincent, Dr. J. M. Carpenter, and co-workers for their work in constructing and operating the phased-rotor machine.

\title{
Force Constants of $\mathrm{N}_{2} \mathrm{O}_{4}$ and $\mathrm{NO}_{2}$ and the Viscosity of the Dissociating System $\mathrm{N}_{2} \mathrm{O}_{4} \rightleftharpoons 2 \mathrm{NO}_{2}$
}

\author{
A. K. Barua and T. K. Rai Dastidar \\ Indian Association for the Cultivation of Science, Calcutta-32, India
}

(Received 6 August 1965)

\begin{abstract}
By analyzing the experimental viscosity of the dissociating system $\mathrm{N}_{2} \mathrm{O}_{4} \rightleftharpoons 2 \mathrm{NO}_{2}$, the force constants of $\mathrm{N}_{2} \mathrm{O}_{4}$ and $\mathrm{NO}_{2}$ have been obtained for the Lennard-Jones (12:6) potential. The calculations made with the force constants thus obtained suggest that, in agreement with theory, chemical reaction has no significant effect on the viscosity of a chemically reacting gas mixture.
\end{abstract}

$\mathbf{T}$ HE knowledge of the transport properties of chemically reacting gas mixtures is of great importance in jet propulsion as these gases exist in the exhaust nozzles of jet engines. In this respect the thermal conductivity of chemically reacting gas mixtures has received greatest attention from the theoretical and the experimental points of view. Experimentally, the dissociating system $\mathrm{N}_{2} \mathrm{O}_{4} \rightleftharpoons 2 \mathrm{NO}_{2}$ has been studied most and the data have been compared with the existing theories. ${ }^{1-3}$ As $\mathrm{N}_{2} \mathrm{O}_{4}$ and $\mathrm{NO}_{2}$ almost always coexist, the force parameters for these molecules are difficult to determine and no reliable direct determination of these have yet been made. However, for a proper comparison between experiment and theory a reliable

\footnotetext{
1 R. S. Brokaw, J. Chem. Phys. 35, 1569 (1961).

2 K. P. Coffin and C. O'Neal, National Advisory Committee for Aeronautics Technical Note 4209, Washington, D. C. (1958). 3 B. N. Srivastava and A. K. Barua, J. Chem. Phys. 35, 329 (1961).
}

set of force parameters of the components of the mixture is essential. In order to calculate the properties of the system $\mathrm{N}_{2} \mathrm{O} \rightleftharpoons 2 \mathrm{NO}_{2}$, Brokaw $^{4}$ obtained the constants for the Lennard-Jones $(12: 6)$ potential for $\mathrm{N}_{2} \mathrm{O}_{4}$ from the boiling-point and liquid-density data and those for $\mathrm{NO}_{2}$ were assumed to be similar to these for $\mathrm{CO}_{2}$ and $\mathrm{N}_{2} \mathrm{O}$. Subsequently, Srivastava, Barua, and Chakraborti ${ }^{5}$ by analyzing the thermal-conductivity data of Barua and Chakraborti ${ }^{6}$ obtained a set of constants for $\mathrm{NO}_{2}$ which were quite different from those obtained in Ref. 4. However, due to the effects of chemical reaction and internal degrees of freedom the force constants determined from the thermal-conductivity data are not very reliable.

\footnotetext{
${ }^{4}$ R. S. Brokaw, National Advisory Committee for Aeronautics Research Memorandum E57K19a, Washington, D. C. (1958).

B. N. Srivastava, A. K. Barua, and P. K. Chakraborti, Trans. Faraday Soc. 59, 2522 (1963).

A. K. Barua and P. K. Chakraborti, J. Chem. Phys. 36, 2817 (1962).
} 